\title{
An Unmanned Helicopter Model Identification Method based on the Immune Particle Swarm Optimization Algorithm
}

\author{
Yang Tingting \\ College of Automation \\ Northwestern Polytechnical University \\ Xi’an, P.R.China \\ yiting@nwpu.edu.cn
}

\author{
Li Aijun \\ College of Automation \\ Northwestern Polytechnical University \\ Xi'an, P.R.China \\ liaijun@nwpu.edu.cn
}

\begin{abstract}
An unmanned helicopter dynamic model identification method based on immune particle swarm optimization (PSO) algorithm is approved in this paper. In order to improve the search efficiency of PSO and avoid the premature convergence, the PSO algorithm is combined with the immune algorithm. The unmanned helicopter model parameters are coded as particle, the error of flight test and math simulation model is objective function, and the dynamic model of unmanned helicopter is identified. The simulation result shows that the method has high identification precision and can realistically reflect the dynamic characteristics.
\end{abstract}

Keywords-model identification; immune PSO; unmanned helicopter

\section{INTRODUCTION}

In recent years, the unmanned helicopter has been widely applied in the fields of industry, agriculture, communications and military. Because of operating distance is limited, automatic / autonomous flight control require precise aircraft dynamics model for the flight control law design.

The helicopter is a highly non-linear, three-axis serious coupling and unstable system. The helicopter model data obtained through the wind tunnel tend to have a lot of inaccuracies. According to the actual flight, flight control law designers often need several years of flight tests to optimize the helicopter control law. This will consume a lot of manpower, material, financial resources and risk is very high $^{[1]}$.

Precise recise unmanned helicopter model is obtained through system identification method for flight control law design, is widely used. In this paper unmanned helicopter is the research object. According to the aircraft test flight attitude response data, immune particle swarm optimization algorithm is used to unmanned helicopter dynamic model identification, and obtains the high precision model.

\section{UNMANNED HELICOPTER MODELING}

In this paper, the Newton - Euler equations are used to build the unmanned helicopter flight dynamics model. As shown in the following formula:

$$
\begin{gathered}
\dot{u}=X_{u} u-g \vartheta+X_{a} a \\
\dot{v}=Y_{v} v-g \phi+Y_{b} b+Y_{p e d} \delta_{p e d} \\
\dot{p}=L_{u} u+L_{v} v+L_{b} b+L_{\omega} w
\end{gathered}
$$

$$
\begin{gathered}
\dot{q}=M_{u} u+M_{v} v+M_{a} a+M_{w} w+M_{c o l} \delta_{c o l} \\
\dot{\phi}=p \\
\dot{\theta}=q \\
\dot{a}=-q-1 / \tau_{f} a+A_{b} b+A_{c} c+A_{\text {lat }} \delta_{\text {lat }}+A_{\text {lon }} \delta_{\text {lon }} \\
\dot{b}=-p-1 / \tau_{f} b+B_{a} a+B_{d} d+B_{\text {lat }} \delta_{\text {lat }}+B_{\text {lon }} \delta_{\text {lon }} \\
\dot{w}=Z_{a} a+Z_{b} b+Z_{w} w+Z_{r} r+Z_{c o l} \delta_{c o l} \\
\dot{r}=N_{v} v+N_{p} p+N_{w} w+N_{r} r+N_{r f b} r_{f b}+N_{p e d} \delta_{p e d}+N_{c o l} \delta_{c o l} \\
\dot{r}_{f b}=K_{r} r+K_{r f b} r_{f b} \\
\dot{c}=-q-1 / \tau_{s} c+C_{l o n} \delta_{l o n} \\
\dot{d}=-p-1 / \tau_{s} d+D_{l a t} \delta_{l a t}
\end{gathered}
$$

Where, $u, v, p, q, \phi, \theta, a, b, w, r, r_{f b}, c, d$ are forward velocity, normal velocity, roll rate, pitch rate, roll, pitch, normal waving angle, horizontal waving angle, lateral velocity, yaw rate, yaw rate feedback, stabilizer stick longitudinal waving angle, stabilizer stick horizontal waving angle. $\delta_{\text {lat }}, \delta_{\text {lon }}, \delta_{\text {ped }}, \delta_{\text {col }}$ are control inputs.

\section{MODEL IDENTIFICATION}

\section{A. Data Processing}

Eliminate some certain errors caused by unmanned helicopter system zero values by using zero calibration method. This zero calibration method is to measure the actual system zero value and then subtract this zero value in the corresponding data.

In addition, the testing data are usually introduced the unreasonable hops, namely outliers, because of the state and environment of the hardware equipment. If incorporating the data, including these outliers, to the model identification, the unmanned helicopter model is definitely inaccurate. Therefore, before model identification, the designer should eliminate these outliers. This paper adopts 7-point secondorder forward differential equation ${ }^{[2]}$.

$$
\begin{aligned}
& \hat{y} 1=(32 y 1+15 y 2+3 y 3-4 y 4-6 y 5-3 y 6+5 y 7) / 42 \\
& \hat{y} 2=(5 y 1+4 y 2+3 y 3+2 y 4+y 5-y 7) / 14 \\
& \hat{y} 3=(y 1+3 y 2+4 y 3+4 y 4+3 y 5+y 6-2 y 7) / 14
\end{aligned}
$$




$$
\begin{aligned}
& \hat{y} 4=(-2 y 1+3 y 2+6 y 3+7 y 4+6 y 5+3 y 6-2 y 7) / 21 \\
& \hat{y} 5=(-2 y 1+y 2+3 y 3+4 y 4+4 y 5+3 y 6+y 7) / 14 \\
& \hat{y} 6=(-y 1+y 2+2 y 4+3 y 5+4 y 6+5 y 7) / 14 \\
& \hat{y} i=\left(5 y_{i-6}-3 y_{i-5}-6 y_{i-4}-4 y_{i-3}+3 y_{i-2}+15 y_{i-1}+32 y_{i}\right) / 42
\end{aligned}
$$

Where, $y i$ are flight testing data, $\hat{y} i$ are testing data from interpolation. If meeting the below equation, these data are regarded as outliers. These outliers should be eliminated and processed smoothly.

$$
|y k-y k|>2.2 \sqrt{\sum_{i=k-6}^{k}(y i-\hat{y} i)^{2} / 6}
$$

\section{B. Model Identification Based on Immune PSO Algorithm}

Particle swarm algorithm finds the global optimum value through updating itself and group optimal values. This algorithm is simple with fast optimization, but it is easy to fall into local optimum. This paper introduces the mutation mechanism of immune mechanisms into PSO algorithm in order to enhance the diversity of the algorithm population. And thus improve the global optimal search ability.

Each particle's position and velocity are calculated by the (17)-(18):

$$
\begin{gathered}
V=w * V+t_{1} * \operatorname{rand}() *(\text { Pbest }-x)+t_{2} * \operatorname{rand}() *(\text { Gbest }-x) \\
P=P+V
\end{gathered}
$$

For particle swarm optimization problems, immune memory and immune regulation of the immune system mechanisms are cited into particle swarm optimization algorithm. In immune particle swarm optimization algorithm, the immune memory mechanism is retained together as the memory cells of the immune system to the antibody of the reaction part of the antigen with the invasion, when the re-invasion of the same type of antigen, the corresponding memory cells are activated to produce large amounts of antibodies. The immunomodulatory mechanism when the antibody and antigen affinity or low concentrations will be promoted, whereas antibody suppressed. The concentration of particles the smaller is the probability of selecting the greater, the greater the concentration of the particles were selected probability is smaller, and at each iteration during groups optimal solution Gbest deemed memory particles are used instead of particle swarmlower fitness particles.

For the particle which not been selected, the reverse flight strategy is adopted.

$$
P=P-V
$$

In order to realize the immune PSO algorithm, density of the particle swarm is defined as (20):

$$
D\left(X_{i}\right)=\frac{1}{\sum_{j=1}^{M+N}\left|F\left(X_{i}\right)-F\left(X_{j}\right)\right|}
$$

$$
P\left(X_{i}\right)=\frac{D^{-1}\left(X_{i}\right)}{\sum_{i=1}^{M+N} D^{-1}\left(X_{i}\right)}=\frac{\sum_{j=1}^{M+N}\left|F\left(X_{i}\right)-F\left(X_{j}\right)\right|}{\sum_{i=1}^{M+N} \sum_{j=1}^{M+N}\left|F\left(X_{i}\right)-F\left(X_{j}\right)\right|}
$$

The unmanned helicopter model is established based on (1)- (13). The parameters

$X_{u}, X_{a}, Y_{v}, Y_{b}, Y_{\text {ped }}, L_{u}, L_{v}, L_{b}, L_{w}, M_{u}, M_{v}, M_{a}, M_{w}, M_{c o l}, A_{b}$, $A_{c}, A_{\text {lat }}, A_{\text {lon }}, B_{a}, B_{d}, B_{\text {lat }}, B_{\text {lon }}, Z_{a}, Z_{b}, Z_{w}, Z_{r}, Z_{c o l}, N_{v}, N_{p}, N_{w}$, $N_{r}, N_{r f b}, N_{p e d}, N_{c o l}, K_{r}, K_{r f b}, C_{l o n}$ which should be selected and optimized should be coded into particles.

The processing of unmanned helicopter model identification based on immune PSO algorithm is:

- Step 1: Initializing particle, initialize the particle swarm in the certain search space according to the physical meaning of optimization parameters.

- $\quad$ Step 2: Dividing the particle swarm into two parts: A and $\mathrm{B}$.

- $\quad$ Step 3: Comparing the processed data $u, v, p, q$, $\phi, \theta, a, b, w, r$ from flight tests with the data $u, v, p, q, \phi, \theta, a, b, w, r$ corresponding to particle model. Regard the sum of the square of these two data's error as the fitness function for optimization.

- $\quad$ Step 4: Comparing the fitness value in part A and renew the Pbest.

- Step 5: Comparing the fitness value in part A and renew the Gbest.

- Step 6: Taking the Gbest into immune memory library. when the optimization stop condition is met, stop the optimization.

- Step 7: Based on the (17)-(18), the position and velocity of the particle in part A are updated.

- Step 8: the position of the particle in part B are updated by (19).

- $\quad$ Step 9: Calculating the density of particle swarm by (20);

- Step 10: Calculating the probability of particle swarm by (21);

- Step 11: Based on the probability, selecting $\mathrm{M}$ particles to form part $\mathrm{A}$, the last particles form the part B;

- $\quad$ Step 12: Replacing the low fitness value particles in the part A with the particles of the immune memory library;

- $\quad$ Step 13: Return to step 3.

The principle of the model identify method could been expressed in the follow Figure 1.

Where: $F\left(X_{i}\right)$ is the fitness of the particle i.

The probability of selected is: 


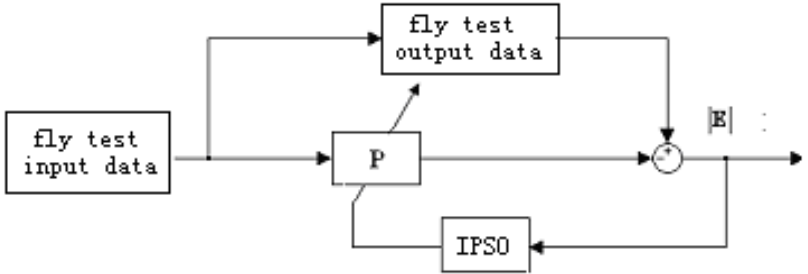

Figure 1. The principle of the model identify

\section{SiMULATION AND RESULTS}

Take CALIBER ZG unmanned helicopter as experimental platform. First, the UAV operator fly the aircraft in hover flight status. Then, the operator command frequency sweep input in pitch, roll, and yaw channels respectively. So the tri-axial velocity, attitude and rate data of the CALIBER helicopter are obtained. The number of the particle swarm is 100 , the number of iterations is select as 200.

\begin{tabular}{|l|c|} 
TABLE I THE PARAMETERS OF CALIBER ZG \\
\hline Fuselage's length & $1585 \mathrm{~mm}$ \\
\hline Fuselage's width & $460 \mathrm{~mm}$ \\
\hline Fuselage's height & $746 \mathrm{~mm}$ \\
\hline Main rotor & $1770 \mathrm{~mm}$ \\
\hline Tail rotor & $270 \mathrm{~mm}$ \\
\hline Weight & $9000 \mathrm{~g}$ \\
\hline
\end{tabular}

Fig. 2 and Fig. 3 show the pitch and roll response. Solid line stand for the flight test curve and dashed line is the response curve of the identification of the model. As can be seen from the figure, a higher degree of agreement reflects in the two curves. The identification model could realistically reflect the actual dynamic characteristics of the helicopter.

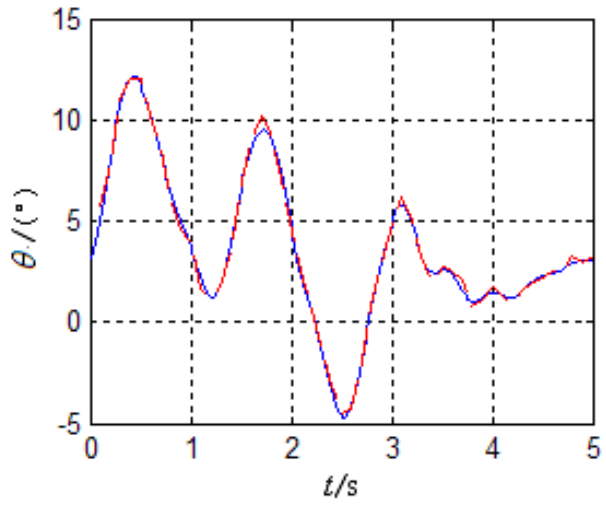

Figure 2. Pitch response

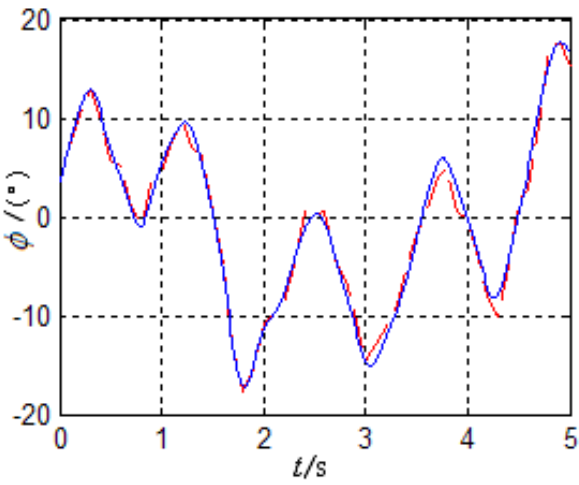

Figure 3. Roll response

\section{CONCLUSION}

An unmanned helicopter dynamic model identification method based on immune particle swarm optimization algorithm is approved in this paper. The algorithm combines the immune memory and the immune regulation. The particle concentration decides whether should restrain the particle or promote the particle, the memory particle is used to instead of the particle with low fitness and the reverse direction flight update strategy of the particle replaces the random initialization. By these methods, the ability of converging to the global optimal solution and the search precision are enhanced. Through improve the global optimization capability of the PSO algorithm; the identification precision of the unmanned helicopter is increased. This research has important value for realizing automatic / autonomous flight control for unmanned helicopter.

\section{REFERENCES}

[1] Sun Tao, Song Yanguo. Study on a Method for Unmanned Helicopter Flight Dynamics Model Identification. Acta Aeronautica et Astronautica Sinica, 2007,28:s14-s18.

[2] Zhao Zhigang, Lv Yisheng. The Preprocessing for Robot Helicopter Model Identification Signals. Journal of Lanzhou Jiaotong University, 2010,29(4): pp.40-43.

[3] Mettler B. Modeling small scale unmanned rotorcraft for advanced flight control design. Pittsburgh: Carnegie Mellon University, 2001.

[4] Bemard M, Mark Tischler. System identification of small size unmanned helicopter dynamics. Journal of the american helicopter society, 2002, 47(1):50-63.

[5] Gao Yin, Xie Shengli. Particle Swarm Optimization Algorithms with Immunity. Computer Engineering and Applications, 2004,6:4-6.

[6] Bemard M. System identification modeling of a model scale helicopter. Reprot CMU-RI-TR-00-03,2000. 